\title{
CINÉTICA E MECANISMO DE OXIDAÇÃO DE UM CONCENTRADO DE ESFALERITA
}

Vitor de Andrade Alvarenga Oliveira ' José Márcio Soares Penna ' Lucas Sanches Magalhães '

\section{Resumo}

A oxidação do concentrado de esfalerita $(\mathrm{ZnS})$ por oxigênio gasoso foi realizada por meio de análise termogravimétrica (TGA). Os produtos de oxidação foram analisados por difratometria de raios $\mathrm{X}$ e microscopia eletrônica de varredura (MEV/EDS) e a formação de sulfato de zinco foi detectada durante o processo de oxidação. A pirita presente no concentrado foi aquecida a uma temperatura mais baixa do que a esfalerita e o mecanismo de oxidação estava de acordo com o mecanismo proposto por Dunn J. G. et. al. (1989). A cinética da oxidação da esfalerita foi avaliada por meio do método de isoconversão de Linhas de Ozawa-Flynn-Wall (OFW), Kissinger-Akahira-Sunose (KAS) e não-linear (MNL) e os resultados sugerem que o processo de oxidação segue um mecanismo complexo. Os valores de energia de ativação encontrados foram superiores a $40 \mathrm{~kJ} / \mathrm{mol}$ na gama completa de conversão sugerindo que a etapa de controle ocorre por meio de reação química.

Palavras-chave: Oxidação da esfalerita; Método de isoconversão; Análise termogravimétrica.

\section{KINETICS AND MECHANISM OF OXIDATION OF A CONCENTRATE OF SPFALERITE}

\begin{abstract}
The oxidation of sphalerite $(\mathrm{ZnS})$ concentrate by gaseous oxygen was carried out by means of thermogravimetric analysis (TGA). The products of oxidation were analyzed by X-ray difractometry and microscopy electronic scanning (MEV/EDS) and the formation of zinc sulphate was detected during the oxidation process. The pyrite present on concentrate was roasted at lower temperature than sphalerite and the mechanism of oxidation was in agreement with the mechanism proposed by Dunn J. G. et. al. (1989). The kinetics of sphalerite oxidation was evaluated by mean of Ozawa-Flynn-Wall (OFW), Kissinger-Akahira-Sunose (KAS) and nonlinear (MNL) isoconversional method and the results suggest that the oxidation process follow a complex mechanism. The values of activation energy founded were higher than $40 \mathrm{~kJ} / \mathrm{mol}$ at full range of conversion and suggest that the controlling step takes place via chemical reaction.
\end{abstract}

Keywords: Sphalerite oxidation; Isoconversional method; Thermogravimetric analysis.

\section{INTRODUÇÃO}

\section{I.I Oxidação de Sulfetos Metálicos}

A extração de zinco do mineral esfalerita $(\mathrm{ZnS})$ é feita, principalmente, por meio do processo RLE (roastingleaching-electrolysis). A reação de ustulação do sulfeto de zinco (Equação I), que é a primeira etapa do processo, pode ser representada pela seguinte equação química:

$$
\mathrm{ZnS}+1,5 \mathrm{O}_{2} \rightarrow \mathrm{ZnO}+\mathrm{SO}_{2} \quad \Delta H^{o}=-450 \mathrm{~kJ} / \mathrm{mol} \mathrm{de} \mathrm{ZnS}
$$

Além de ser uma reação exotérmica, a utilização de temperaturas na faixa de $600^{\circ} \mathrm{C}-1000^{\circ} \mathrm{C}$, para a ustulação de sulfetos minerais, se deve à questões cinéticas e não termodinâmicas. Vale destacar que a esfalerita entra no reator de ustulação como um concentrado e não como fase pura e, por isso, a presença de diferentes minerais contaminantes como pirita $\left(\mathrm{FeS}_{2}\right)$, galena $(\mathrm{PbS})$ e sílica $\left(\mathrm{SiO}_{2}\right)$ podem promover a formação de fases mais refratárias contendo $\mathrm{Zn}$ e, dessa forma, promoverem um menor rendimento na etapa de lixiviação. Como exemplo, a literatura específica relata que $o$ aumento na quantidade de marmatita ( $\mathrm{nZnS} . \mathrm{mFeS})$

'Departamento de Engenharia Metalúrgica, Universidade Federal de Ouro Preto - UFOP, Campus Morro do Cruzeiro, Ouro Preto, MG, Brasil. E-mail:victor@demet.em.ufop.br

2176-1523 (C) 2019 Associação Brasileira de Metalurgia, Materiais e Mineração. Publicado pela ABM. Este é um artigo de acesso aberto distribuído sob os termos da licença Creative Commons CC BY-NC-ND (Attribution-NonCommercial-NoDerivs) - https:// creativecommons.org/licenses/by-nc-nd/4.0\%. 
presente em um concentrado sulfetado de zinco aumenta a formação de ferrita de zinco que, consequentemente, reduz a eficiência da etapa de lixiviação $[1,2]$.

A grande perda de massa e a grande liberação de energia associadas às reações e aos mecanismos químicos de oxidação de minerais sulfetados fazem com que os métodos termoanalíticos tenham especial importância para o entendimento dessas reações e, devido à grande complexidade dos mecanismos químicos envolvidos nas reações de ustulação de sulfetos minerais, diferenças significativas entre os resultados encontrados e as interpretações dadas aos diferentes eventos, por diferentes autores, para o mesmo mineral, são comuns $[3,4]$.

A pressão parcial do agente oxidante possui extrema importância quando se quer prever as fases que estarão presentes após a oxidação do mineral sulfetado. Para o caso da pirita, vários autores relatam que o emprego de condições fortemente oxidantes levará a formação de $\mathrm{Fe}_{2} \mathrm{O}_{3}$. No entanto, alguns autores relatam a formação de espécies menos oxidadas como $\mathrm{Fe}_{3} \mathrm{O}_{4}$ e/ou $\mathrm{FeO}$ em estudos onde utilizou-se também de uma atmosfera fortemente oxidante [5].

A formação de uma camada de óxido na superfície do mineral sulfetado durante a oxidação do sulfeto metálico pode promover condições para a formação de diversas reações sólido-sólido. Além disso, grandes pressões de $\mathrm{SO}_{2}$ ou $\mathrm{SO}_{3}$ podem ser geradas na interface óxido-sulfeto, devido a oxidação ou decomposição do núcleo sulfetado e a formação de sulfato tornar-se termodinamicamente favorável, sendo assim, normalmente temos uma camada mais externa de óxido, uma camada intermediária de sulfato e uma camada interna de sulfeto, o que justifica o emprego do modelo do núcleo não reagido para o estudo cinético dessas reações. Por outro lado, alguns autores mostram evidências de que a reação de oxidação de sulfetos ocorre através da oxidação preferencial de alguns planos cristalinos da partícula e não segundo o modelo do núcleo não reagido [I,5-7].

Dentro deste contexto, o objetivo deste trabalho foi realizar um estudo da cinética e dos mecanismos que envolvem a oxidação de um concentrado de esfalerita, determinando assim a etapa controladora do processo bem como a energia de ativação segundo os diferentes métodos integrais que serão utilizados.

\section{I.2 Análise Cinética - Método Isoconversional}

O princípio isoconversional estabelece que para uma razão $\beta$ de aquecimento, temos:

$$
\beta \frac{d \alpha}{d T} \equiv A \exp \left(\frac{-E}{R T}\right) f(\alpha)
$$

onde $\mathrm{A}$ e $\mathrm{E}$ são o fator pré-exponencial e a energia de ativação, respectivamente, $\mathrm{f}(\alpha)$ é a função conversão, $\mathrm{R}$ é a constante dos gases, T é a temperatura, té o tempo e $\alpha$ é a extensão da conversão. Para uma determinada conversão, a Equação 2 pode ser escrita como:

$$
\ln \left(\beta \frac{d \alpha}{d T}\right)_{\alpha}=\ln \left(A_{\alpha} f(\alpha)\right)-\frac{E_{\alpha}}{R T_{\alpha}}
$$

Onde o símbolo $\alpha$ indica que a equação é aplicada para um determinado valor de conversão. A Equação 3 mostra que para experimentos feitos com diferentes taxas de aquecimento, um gráfico de $\ln \left(\beta \frac{d \alpha}{d T}\right)_{\alpha}$ como função de $\mathrm{T}^{-1}$ será uma reta com coeficiente angular igual a $-\frac{E}{R}$. Esse
método cinético é conhecido como método diferencial de Friedman e, para que seja aplicado, a diferenciação das curvas $\alpha$ vs T deve ser feita. $O$ principio isoconversional pode também ser aplicado à integral (Equação 4) e, nesse caso, o método utilizado é denominado método integral e é baseado na integração da Equação 2, isto é:

$$
g(\alpha) \equiv \frac{A}{\beta} \int_{0}^{T_{\alpha}} \exp \left(\frac{-E_{a}}{R T}\right) d t=\frac{A}{\beta} I(E, T)
$$

a integral $\mathrm{I}(\mathrm{E}, \mathrm{T})$ não possui solução analítica e normalmente é resolvida a partir de métodos numéricos. Duas expressões que podem ser utilizadas como soluções aproximadas para a integral I(E,T) são as expressões de Ozawa-Flynn-Wall (OFW) (Equação 5) e Kissinger-Akahira-Sunose (KAS) (Equação 6).

$$
\begin{aligned}
& -\ln \beta=1.0516\left(\frac{E_{a}}{R T}\right)-A^{\prime} \\
& -\ln \left(\frac{\beta}{T^{2}}\right)=\frac{E_{a}}{R T}-\ln \left(\frac{A^{\prime \prime} R}{E_{a}}\right)
\end{aligned}
$$

Nesse caso, um gráfico de $-\ln \beta$ vs $1 / T$ ou $-\ln \left(\beta / T^{2}\right)$ vs $1 / T$ será uma linha reta a partir da qual a energia de ativação pode ser calculada [8].

Um procedimento não linear (MNL) também foi empregado neste trabalho onde se utilizou da aproximação de Senum-Yang [9] (Equação 7) para a solução da integral I(E,T)

$$
I(E, T) \cong \frac{\exp (-x)}{x} \cdot \frac{\left(x^{2}+10 x+18\right)}{\left(x^{3}+12 x^{2}+36 x+24\right)}
$$

Esse procedimento estabelece (Equações 8 e 9) que, assumindo que o modelo reacional é independente da taxa de aquecimento, temos que:

$$
\begin{aligned}
& \sum_{i=1}^{n} \sum_{i \neq j}^{n}\left[I\left(E_{\alpha}, T_{\alpha, i}\right) \beta_{j}\right] /\left[I\left(E_{\alpha}, T_{\alpha, j}\right) \beta_{i}\right]=n(1-n) \\
& n(1-n)-\sum_{i=1}^{n} \sum_{i \neq j}^{n}\left[I\left(E_{\alpha}, T_{\alpha, i}\right) \beta_{j}\right] /\left[I\left(E_{\alpha}, T_{\alpha, j}\right) \beta_{i}\right]=\text { mínimo }
\end{aligned}
$$

Onde $\beta \mathrm{i}$ e $\beta \mathrm{j}(\mathrm{i}=\mathrm{I}, \ldots, \mathrm{n})$ são as taxas de aquecimento utilizadas nos experimentos i e j. Nesse procedimento, o valor para a energia de ativação, para um determinado grau de conversão, é aquele que satisfaz a condição de mínimo para a Equação 9 [10]. 


\section{MATERIAIS E MÉTODOS}

O concentrado de esfalerita utilizado foi classificado e todas as análises foram feitas com a fração granulométrica entre 100 e 150 mesh.

\section{I Análise Química}

O método para quantificar os elementos químicos presentes no concentrado foi digestão ácida, utilizando-se ácido clorídrico, nítrico e fluorídrico, sob aquecimento. Os teores de determinados elementos presentes ( $\mathrm{Zn}, \mathrm{S}, \mathrm{Fe}, \mathrm{Cu}, \mathrm{Si}, \mathrm{Pb}, \mathrm{Mn}, \mathrm{Cd}, \mathrm{Al}$ e As) foram analisados por espectrometria de emissão atômica com fonte de plasma indutivamente acoplado (ICP-OES), marca Varian modelo 725-ES.

\subsection{Termogravimetria (TGA) e Calorimetria Exploratória Diferencial (DSC)}

Os ensaios de oxidação foram feitos utilizando-se de uma taxa de aquecimento de $10^{\circ} \mathrm{C} / \mathrm{min}$ e a faixa de temperatura de $30^{\circ} \mathrm{C}$ até $1000^{\circ} \mathrm{C}$. A fase oxidante utilizada foi $\mathrm{O}_{2}(99,99 \%)$ a uma vazão de $60 \mathrm{ml} / \mathrm{min}$. Esse valor de vazão foi escolhido pois acima de $50 \mathrm{ml} / \mathrm{min}$ a curva TGA não foi mais influenciada por esse parâmetro. Para o estudo cinético, as seguintes taxas de aquecimento foram utilizadas, $5^{\circ} \mathrm{C} / \mathrm{min}, 10^{\circ} \mathrm{C} / \mathrm{min}, 15^{\circ} \mathrm{C} / \mathrm{min}$ e $20^{\circ} \mathrm{C} / \mathrm{min}$. Os experimentos foram feitos em um equipamento Mettler Toledo modelo TGA/DSC I Star System.

Após a identificação de todos os eventos presentes nas curvas TGA e DSC, seis rampas de aquecimento foram feitas nas mesmas condições estabelecidas na seção 2.2 , porém, nesses estudos as temperaturas finais dos experimentos foram de $400^{\circ} \mathrm{C}, 500^{\circ} \mathrm{C}, 600^{\circ} \mathrm{C}, 700^{\circ} \mathrm{C}$, $800^{\circ} \mathrm{C}$ e $1000^{\circ} \mathrm{C}$. Após o sistema atingir a temperatura final ele foi mantido nessa temperatura por 10 minutos e as amostras geradas foram metalizadas com ouro e analisadas por meio da técnica de microscopia eletrônica de varredura (MEV) acoplada a um espectrômetro de energia dispersiva (EDS).

\subsection{Microscopia Eletrônica de Varredura (MEV) e Espectrometria de Energia Dispersiva (EDS)}

Para a análise das amostras, utilizou-se um equipamento da marca Tescan modelo VEGA 3 LMH acoplado a um EDS da marca Oxford INCA x-act modelo 5I-ADD0007.

\subsection{Difratometria de Raios-X (XRD)}

A identificação das fases presentes na matéria prima e nas amostras geradas nos experimentos da seção 2.2 foi feita utilizando-se de um difratômetro de raios- $X$ da marca Shimadzu modelo XRD-6I00.

\subsection{Análise Cinética}

O estudo cinético da reação de ustulação do concentrado de esfalerita foi feito utilizando-se do método diferencial de Friedman e dos métodos integrais de OFW, KAS e MNL.

\section{RESULTADOS E DISCUSSÃO}

\section{I Caracterização da Amostra Inicial}

A partir da digestão ácida do concentrado de esfalerita, a seguinte composição química foi encontrada: $\mathrm{Zn}-54,1 \%$, $\mathrm{S}-33,06 \%, \mathrm{Fe}-7,28 \%, \mathrm{Cu}-0,91 \%, \mathrm{Si}-0,79, \mathrm{~Pb} 0,46 \%$, $\mathrm{Mn}-0,46 \%, \mathrm{Cd}-0,27 \%, \mathrm{Al}-0,18 \%$, As -0, I2\%. Já na Figura I apresenta-se uma imagem de microscopia eletrônica bem como os resultados de EDS obtidos. Os resultados indicam a presença de alguns elementos como $\mathrm{Cu}$, Si e Al que estão em fases minoritárias. A presença desses elementos é comum nos concentrados de esfalerita e, no presente estudo, focaremos apenas no comportamento do $S, F e, Z n$ e O.

Observa-se na Figura I que o ferro está presente no concentrado como pirita e também substituindo o $\mathrm{Zn}$ na rede da esfalerita, o zinco está presente principalmente como esfalerita e o teor de oxigênio nesse mineral é muito baixo, por outro lado, o oxigênio está mais concentrado nas partículas que possuem mais ferro. É possível observar também que a concentração de enxofre é maior nas partículas com maior concentração de zinco e menor nas partículas com maior concentração de ferro, isso se deve à maior estabilidade do sulfeto de zinco. Além disso, pode-se atribuir a maior concentração de oxigênio na pirita à oxidação natural preferencial desse mineral frente à esfalerita. Observa-se também que apesar da substituição do zinco pelo ferro nas partículas de esfalerita o contrário não é verdade nas partículas de pirita. Finalmente, observa-se na Figura I (partícula $\mathrm{A}$ mais à esquerda) a presença de um grão de esfalerita dentro de uma partícula de pirita, essa imagem mostra um grão de esfalerita não liberado e, confirmando o que foi dito anteriormente, a pirita encontra-se mais oxidada.

Também observa-se na Figura I dois tipos principais de partículas, uma densa e compacta (A e B) (característica de minerais sulfetados) e outra porosa e mais clara $(X)$, os resultados de MEV-EDS mostram que as partículas porosas são aglomerados de pequenas partículas de minerais sulfetados com composição química muito parecida com a do concentrado como um todo.

Observa-se na Figura 2 as curvas TGA e DSC para o concentrado estudado, como pode ser visto, a linha base não estabiliza mesmo nas temperaturas em que nenhum evento é detectado na curva DSC $\left(<350^{\circ} \mathrm{C}\right)$ isso pode ser atribuído à oxidação do concentrado sulfetado que é espontânea mesmo em baixas temperaturas. Acima de $350^{\circ} \mathrm{C}$, cinco eventos térmicos foram identificados e denominados $A, B, C, D$ e $E$ na curva termogravimétrica. 

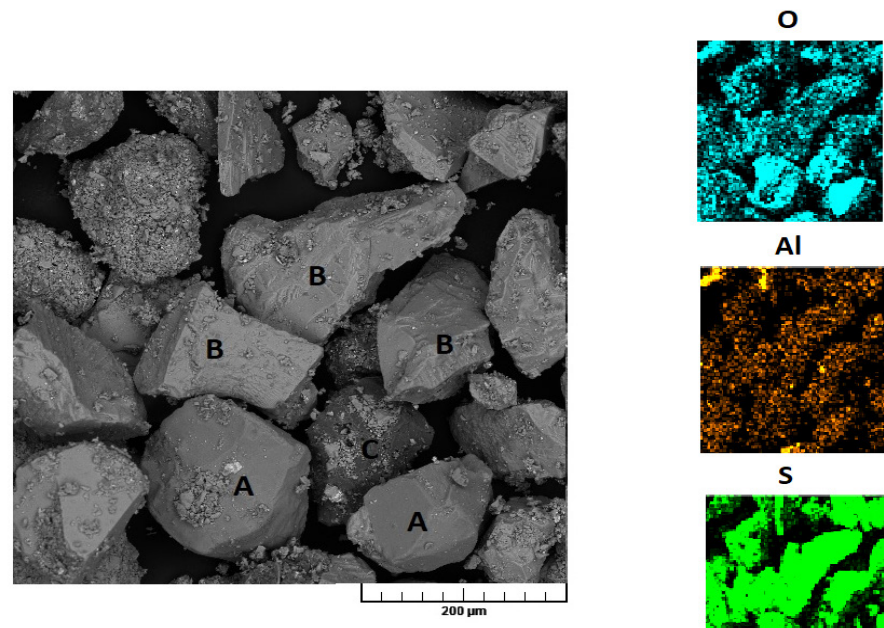

Al

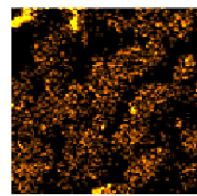

S

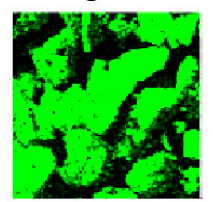

$\mathrm{Fe}$

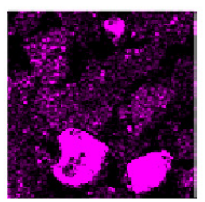

$\mathrm{Cu}$

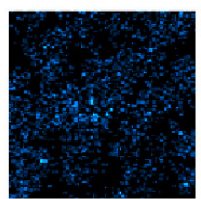

Zn

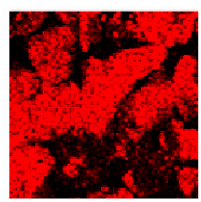

Si

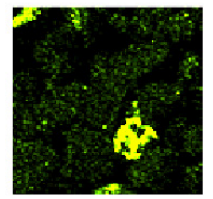

Figura I. Resultados de microscopia eletrônica e mapeamento por espectrometria de energia dispersiva do concentrado de esfalerita (A) FeS 2 - (B) $\mathrm{ZnS}-(\mathrm{C}) \mathrm{SiO}_{2}$.

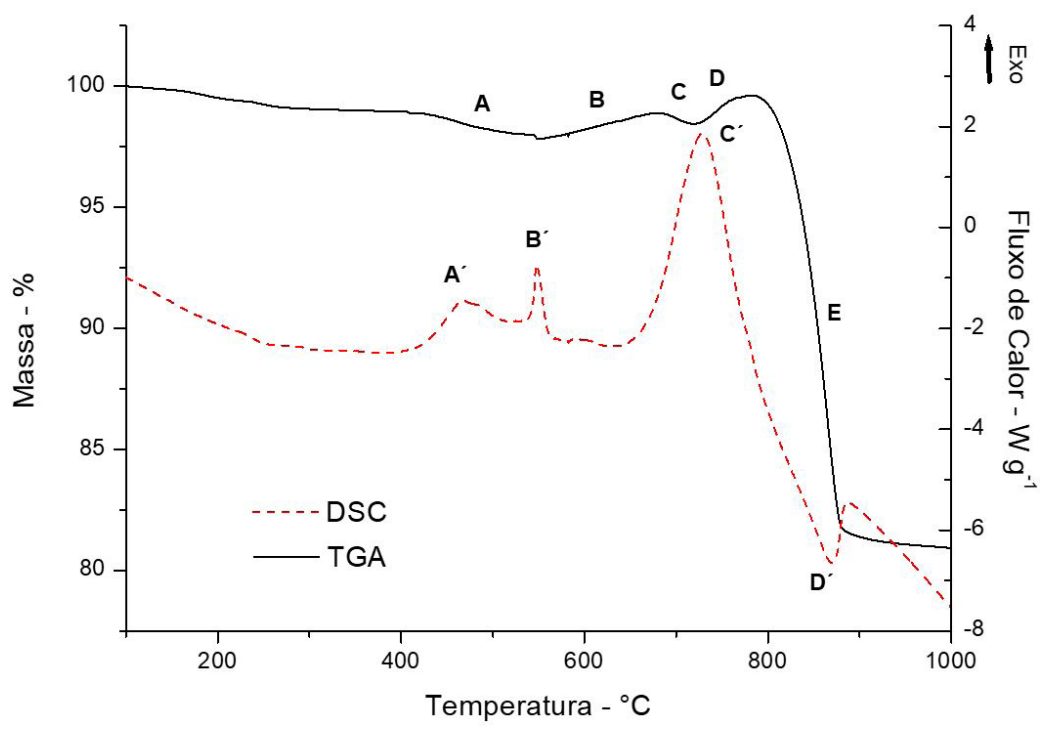

Figura 2. Curvas TGA e DSC para o concentrado de esfalerita. (A) Ustulação da pirita; (B) Formação de FeSO; (C) Ustulação da esfalerita; (D) Formação de $\mathrm{ZnSO}_{4}$; (E) Decomposição do ZnSO4 A`: Formação de camada de óxido no exterior da partícula de pirita. $\mathrm{B}^{\prime}$ :Ignição da partícula de pirita devido ao rompimento da camada de óxido formada $C^{\prime}$ : Evento exotérmico referente a ustulação da esfalerita. $D^{\prime}$ : Decomposição do $\mathrm{ZnSO}_{4}$.

Na curva de DSC quatro eventos foram identificados, sendo três exotérmicos $\left(A^{\prime}, B^{\prime}\right.$ e $\left.C^{\prime}\right)$ e um endotérmico $\left(D^{\prime}\right)$.

A reação de ustulação da esfalerita (Equação 10) é dada por:

$$
\mathrm{ZnS}+1,5 \mathrm{O}_{2} \rightarrow \mathrm{ZnO}+\mathrm{SO}_{2}
$$

a perda de massa esperada para o sistema trabalhando como fase pura seria de $16,49 \%$ e o valor experimental obtido foi de 18,97\%. Apesar da diferença entre o valor teórico e o valor experimental, uma pequena diferença já era esperada uma vez que os resultados são para um concentrado e não para uma fase pura.

Atribuiu-se ao evento A, a ustulação da pirita $\left(\mathrm{FeS}_{2}\right)$ (Equações II, I2 e I3) segundo as seguintes reações:

$$
\mathrm{FeS}_{2}(s)+(1-x) \mathrm{O}_{2}(g) \rightarrow \mathrm{FeS}_{(1+x)}(s)+(1-x) \mathrm{SO}_{2}(g)
$$




$$
\begin{aligned}
& 3 \mathrm{FeS}_{(1+x)}(\mathrm{s})+(3 x+5) \mathrm{O}_{2}(g) \rightarrow \mathrm{Fe}_{3} \mathrm{O}_{4}(s)+(3+3 x) \mathrm{SO}_{2}(g) \\
& 3 \mathrm{FeS}_{2}(\mathrm{~s})+8 \mathrm{O}_{2}(\mathrm{~g}) \rightarrow \mathrm{Fe}_{3} \mathrm{O}_{4}+6 \mathrm{SO}_{2}(g)
\end{aligned}
$$

Todas essas reações são exotérmicas, o que está de acordo com os resultados de DSC, além disso, o mesmo valor de temperatura de reação de $400^{\circ} \mathrm{C}$ levando à formação de FeS foi encontrado por outros autores [4]. Observa-se que estão associados ao evento A dois eventos exotérmicos na curva DSC, segundo o mecanismo de oxidação proposto por J. G. Dunn e B. H. O'Connor [5], durante a oxidação de amostras de pirita com tamanho de partícula entre 90 e $125 \mu \mathrm{m}$, uma camada de óxido é formada no exterior da partícula (evento $A^{\prime}$ ), essa camada impede a difusão do oxigênio para o interior da partícula e, consequentemente, o núcleo da partícula reage como se a amostra estivesse submetida a uma atmosfera inerte [5]. Sendo assim, a pirolise da pirita (Equação 14) ocorre segundo a seguinte equação química:

$$
\mathrm{FeS}_{2} \rightarrow \mathrm{FeS}_{(1+x)}+0,5 \mathrm{~S}_{2}
$$

Com o aumento da temperatura, a pressão de $\mathrm{S}_{2}$ no interior da partícula aumenta e a camada de óxido formada é rompida, a exposição do núcleo não oxidado à altas temperaturas provoca a ignição da partícula de pirita e, consequentemente, o aparecimento do segundo evento exotérmico (evento $B^{\prime}$ ) na curva DSC.

O ganho de massa, evento $B$ na curva termogravimétrica, foi atribuído à formação de sulfato de ferro a partir dos diferentes óxidos de ferro formados e ao aumento no estado de oxidação dos óxidos de ferro presentes na amostra, a Equação I 5 mostra uma das possíveis reações de formação de sulfato. Destaca-se aqui que as reações de formação de $\mathrm{FeSO}_{4}$ e $\mathrm{Fe}_{3}\left(\mathrm{SO}_{4}\right)_{2}$, a partir de diferentes óxidos de ferro, são todas termodinamicamente favoráveis na temperatura em que ocorre o evento $B$ :

$$
\mathrm{Fe}_{2} \mathrm{O}_{3}+0,5 \mathrm{O}_{2}+2 \mathrm{SO}_{2} \rightarrow 2 \mathrm{FeSO}_{4}
$$

Ao atingir a temperatura de $680^{\circ} \mathrm{C}$, evento $\mathrm{C}$, a esfalerita é ustulada (Equação 16) e, como pode ser visto, um evento exotérmico é observado na curva DSC ( $\left.C^{\prime}\right)$. A ustulação da esfalerita aumenta a pressão de $\mathrm{SO}_{2}$ dentro do leito e, consequentemente, a formação de $\mathrm{ZnSO}_{4}$ (Equação 17) torna-se termodinamicamente favorável, justificando assim o ganho de massa representado pelo evento $D$.

$$
\begin{aligned}
& \mathrm{ZnS}_{(s)}+3 / 2 \mathrm{O}_{2(g)}=\mathrm{ZnO} \mathrm{O}_{(s)}+\mathrm{SO}_{2(g)} \\
& \mathrm{ZnO}(\mathrm{s})+\mathrm{SO}_{2}(\mathrm{~g})+\frac{1}{2} \mathrm{O}_{2}(g) \leftrightarrow \mathrm{ZnSO}_{4}(s)
\end{aligned}
$$

Pode-se destacar aqui que a reação de formação de $\mathrm{SO}_{3}$ (Equação 18) é exotérmica e, por isso, em altas temperaturas o equilíbrio é deslocado para a esquerda de forma que nem todo o $\mathrm{SO}_{2}$ produzido na etapa de ustulação é convertido $\mathrm{SO}_{3}$, reagente necessário para a formação do sulfato. A presença de diferentes óxidos metálicos pode catalisar a reação de formação de $\mathrm{SO}_{3}$ e, dessa forma, promover uma maior conversão de óxido para sulfato em diferentes amostras minerais [6].

$$
\mathrm{SO}_{2}(g)+\mathrm{O}_{2}(g) \rightarrow \mathrm{SO}_{3}(g)
$$

Atribuiu-se a perda de massa $\mathrm{E}$, a reação de decomposição do sulfato de zinco formado (Equação 19). Como mostra a curva DSC, essa reação é endotérmica (evento D') e, de acordo com os dados termodinâmicos, a decomposição do sulfato de zinco em óxido de zinco ocorre em temperaturas superiores a $955^{\circ} \mathrm{C}$ [6].

$$
\mathrm{ZnSO}_{4}(s) \rightarrow \mathrm{ZnO}(s)+\mathrm{SO}_{2}(\mathrm{~g})+0,5 \mathrm{O}_{2}
$$

Os sólidos formados antes e depois de cada evento presente na curva TGA foram analisados utilizando-se das técnicas de difratometria de raios- $X$ e microscopia eletrônica de varredura. Os difratogramas de raios- $X$ apresentados na Figura 3 mostram que os picos de difração da esfalerita estão presentes naqueles obtidos nos ensaios feitos em temperaturas inferiores a $800^{\circ} \mathrm{C}$, o que justifica as atribuiçõos feitas aos eventos térmicos na curva TGA, e que a formação de sulfato de zinco e óxido de zinco ocorrem simultaneamente. Destaca-se aqui que os resultados indicam que as etapas de oxidação da pirita são diferentes das etapas de oxidação da esfalerita, isto é, o evento exotérmico de oxidação da esfalerita ocorre durante um período de tempo maior e, sendo assim, mesmo em temperaturas superiores à temperatura de início de oxidação, é possível detectar a presença de esfalerita na amostra (Figura $3\left(700^{\circ} \mathrm{C}\right)$ ). É possível observar também que a $400^{\circ} \mathrm{C}$ a fase amorfa presente no concentrado foi oxidada, isto é, o difratograma mostrado na Figura 3 a $400^{\circ} \mathrm{C}$ mostra um material mais cristalino do que o difratograma obtido para a amostra in natura (resultado não apresentado). Por isso, acredita-se que a oxidação dessa fase não cristalina faz com que a linha base da curva TGA (Figura 2) não estabilize.

Como a pirita está presente no concentrado como fase minoritária, as fases minerais formadas durante os eventos térmicos de oxidação desse mineral não foram identificadas nos difratogramas de raios- $X$ e a única fase mineral contendo ferro que pôde ser detectada foi a ferríta de zinco que parece ser formada logo assim que a oxidação da esfalerita se inicia, isto é, a formação de ferríta de zinco pode ser atribuída, principalmente, ao ferro presente na rede da esfalerita.

$\mathrm{Na}$ Figura 4 apresentam-se os resultados de MEV-EDS para o concentrado de esfalerita ustulado a $500^{\circ} \mathrm{C}$, é possível observar que o oxigênio está mais concentrado na pirita e que a oxidação da esfalerita é bem menor que a da pirita. Pode-se observar um grão de esfalerita no interior da partícula de pirita na diagonal inferior esquerda da Figura $4\left(500^{\circ} \mathrm{C}\right)$, essa imagem mostra que a esfalerita ainda não foi oxidada. Como pode ser visto, o grão de pirita ainda possui enxofre o que dá suporte à formação de uma fase mais pobre em enxofre $\left(\mathrm{FeS}_{1+x}\right)$ durante a ustulação da pirita (Equação I4), 


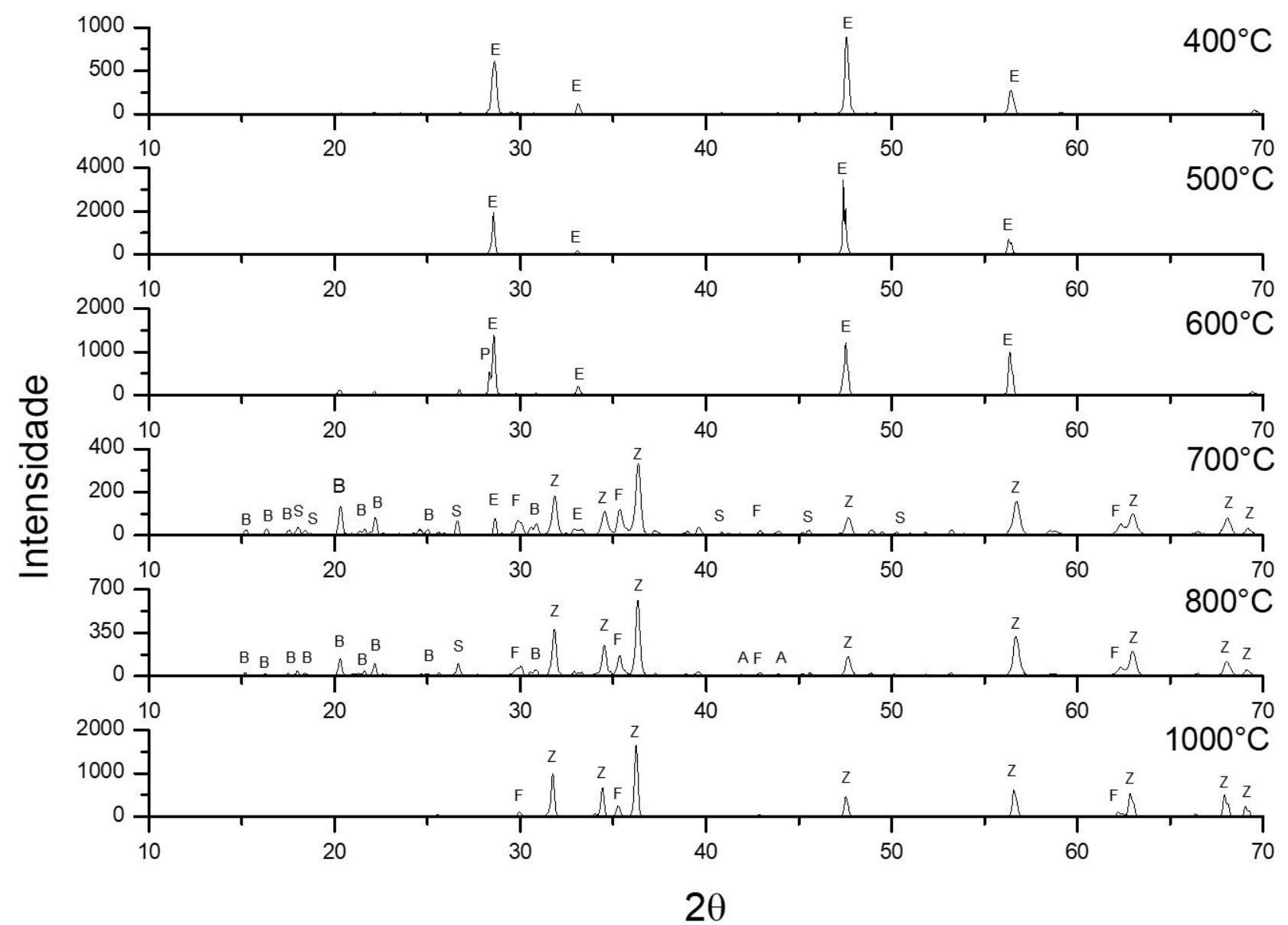

Figura 3. Difratogramas de raios-X para os produtos da ustulação obtidos em diferentes temperaturas ( $E$ - esfalerita; $P$ - pirita; $S$ - sulfato de zinco hidratado; Z - zincita; F - ferrita de zinco; B - bianchita; A - sulfato de zinco). Varredura em ângulo de $8^{\circ}$ até $80^{\circ}$. Velocidade do goniômetro: $2^{\circ}$ por minuto.

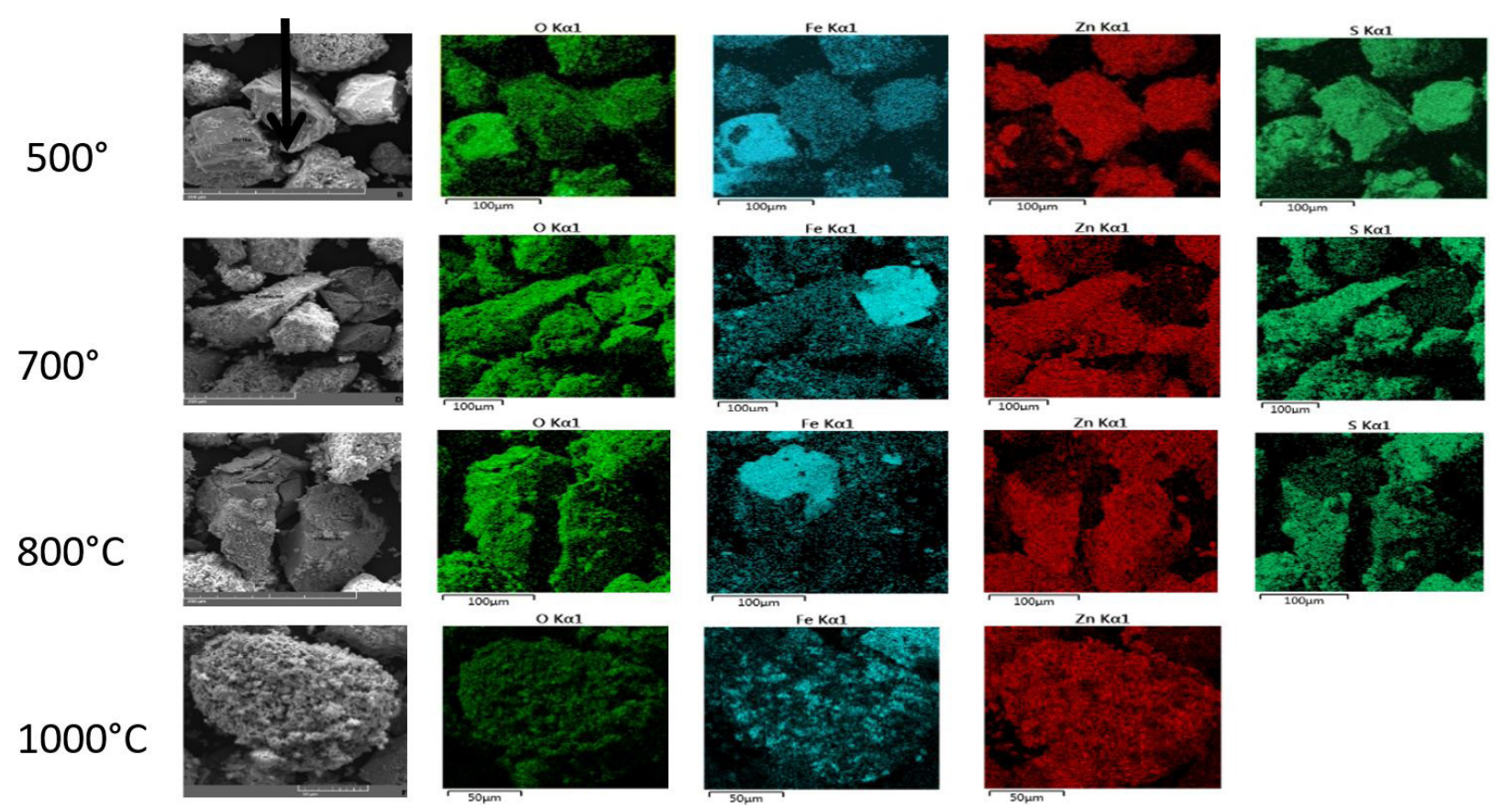

Figura 4. Microscopia eletrônica e mapeamento por espectrometria de energia dispersiva do concentrado de esfalerita ustulado a $500^{\circ} \mathrm{C}, 700^{\circ} \mathrm{C}, 800^{\circ} \mathrm{C}$ e $1000^{\circ} \mathrm{C}$. 
observa-se também que a partícula de pirita deficiente em enxofre é ainda densa e compacta o que é uma característica dos minerais sulfetados.

A partir dos resultados de MEV-EDS para o concentrado de esfalerita ustulado a $600^{\circ} \mathrm{C}$ pôde-se observar que toda a pirita foi ustulada.

Os resultados de MEV-EDS para o concentrado de esfalerita ustulado a $700^{\circ} \mathrm{C}$ mostram que, nessa temperatura, toda a fase contendo ferro foi ustulada e tornou-se mais porosa. Pode-se observar também que apesar do aumento no teor de oxigênio nas partículas de esfalerita, esta ainda contém muito enxofre e, morfologicamente, ainda é uma partícula densa e compacta. A morfologia das partículas de pirita ustuladas (Figura 4) está de acordo com o mecanismo de oxidação proposto Dunn J. G. et. al. [5]. Observa-se também na Figura 4 que a partícula reagente foi fraturada, isto é, o aumento na pressão de $\mathrm{S}_{2}$ no interior da mesma promoveu uma fragmentação da mesma [5].

Os resultados de MEV-EDS para o concentrado de esfalerita ustulado a $800^{\circ} \mathrm{C}$ mostram que a amostra de esfalerita ainda possui muito enxofre, mesmo estando acima da temperatura do ponto de ignição desse mineral, acredita-se que a presença de enxofre na amostra se deve à formação de $\mathrm{ZnSO}_{4}$ segundo a reação 16 , isso é confirmado pelo difratograma de raios- $X$ da amostra.

Os resultados de MEV-EDS para o concentrado de esfalerita ustulado a $1000^{\circ} \mathrm{C}$ mostram que nessa temperatura toda a mostra foi ustulada, observa-se que enxofre não é mais detectado na amostra. Além disso, pode-se observar que a morfologia do ustulado de esfalerita é consideravelmente diferente da morfologia do ustulado de pirita, isto é, o produto da ustulação da esfalerita é muito poroso e as partículas de pirita não estão fragmentadas após o processo. Sendo assim, pode-se concluir que durante a ustulação da esfalerita o óxido formado na superfície da partícula é poroso e, por isso, não impede a difusão do oxigênio para o interior da partícula e nem dos produtos gasosos para o exterior da mesma, consequentemente, o evento exotérmico na curva DSC é mais largo, sugerindo que a partícula não entrou em ignição e sim foi oxidada de fora para dentro continuamente.

A cinética de oxidação da esfalerita foi estudada utilizando-se dos métodos isoconversionais diferencial e integral. A utilização do princípio isoconversional para a análise cinética de processos heterogêneos é bastante vantajosa, pois não é necessário o conhecimento prévio do modelo cinético para que a análise dos dados seja feita. Além disso, esse método também permite determinar o valor da energia de ativação aparente para diferentes graus de conversão. Para processos químicos heterogêneos, isso é de grande importância, pois mudanças na etapa controladora do processo durante o desenvolvimento da reação são comuns, isto é, a etapa lenta de uma reação heterogênea pode ser uma etapa química no início do processo, quando não temos a presença de produtos de reação sólidos aderidos à superfície da partícula, e tornar-se uma etapa controladora de transporte a medida que uma camada de produto sólido de reação é formada na superfície da partícula. A utilização de um método cinético que determine o valor da energia de ativação para um determinado grau de conversão torna-se bastante útil, pois, como se sabe, processos controlados por etapas de transporte possuem valores de energia de ativação muito menores do que aqueles onde a etapa controladora é uma etapa química. Dessa forma, o método isoconversional pode ser utilizado para determinar se uma variação de etapa de controle químico para controle por transporte está ocorrendo durante um processo [II, I2].

A partir da curva DTG para o evento $\mathrm{E}$ em diferentes taxas de aquecimento $\left(5,10^{\circ} \mathrm{C} / \mathrm{min}, 15^{\circ} \mathrm{C} / \mathrm{min}\right.$ e $\left.20^{\circ} \mathrm{C} / \mathrm{min}\right)$ foi possível observar que o aumento na taxa de aquecimento promoveu um deslocamento do evento térmico para maiores temperaturas. A energia de ativação aparente da reação foi determinada por meio do método diferencial de Friedman e dos métodos integrais OFW, KAS e MNL. Uma vez que, a energia de ativação variou muito com o grau de conversão e a temperatura da amostra assumiu valores muito diferentes dos valores da temperatura de referência, isto é, a oxidação de sulfetos metálicos é muito exotérmica, o método diferencial de Friedman não foi utilizado para a análise cinética dos dados experimentais [13]. A Figura 5 mostra a curva de $E \alpha$ como função de $\alpha$ para os diferentes métodos integrais utilizados nesse trabalho.

Os valores de energia de ativação encontrados para os diferentes métodos integrais são muito próximos e não diferem entre si em mais do que $2 \%$. Como esperado, os valores encontrados para a energia de ativação aparente mostram que o sistema em estudo é um sistema complexo e, por isso, variações nos valores da energia de ativação aparente podem ser observados para diferentes graus de conversão. No entanto, mesmo para grandes valores de conversão $(\alpha>0,7)$ a presença da camada de produtos sólidos na superfície da partícula não fez com que a etapa lenta do processo se tornasse uma etapa de transporte, pois os valores de energia de ativação encontrados foram muito maiores que $40 \mathrm{~kJ} / \mathrm{mol}$ [ 12$]$. Sendo assim, acredita-se que nas

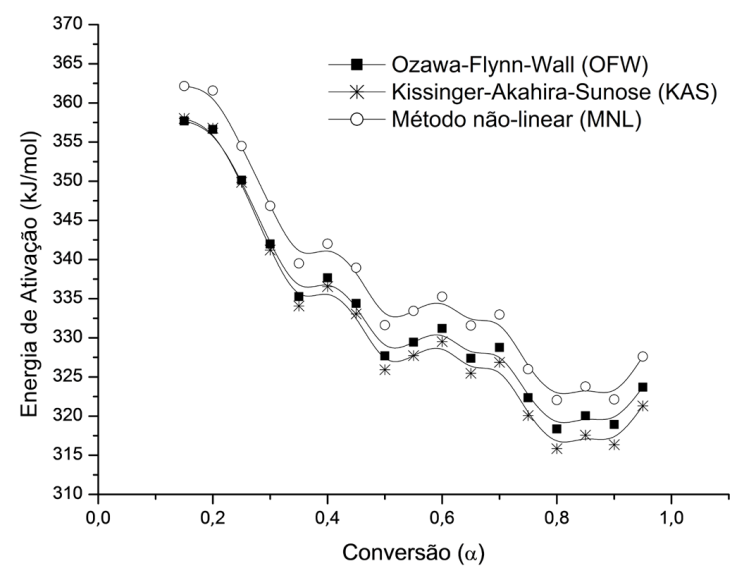

Figura 5. Energia de ativação aparente como uma função do grau de conversão do concentrado de esfalerita segundo os diferentes métodos integrais utilizados nesse trabalho. 
condições experimentais empregadas, a camada de óxido formada durante a reação de ustulação foi suficientemente porosa para não impedir a difusão das espécies gasosas para a superfície da partícula não reagida, nem dos produtos de reação formados para fora da partícula e, por isso, a etapa lenta é uma etapa química durante todo o processo de oxidação.

\section{CONCLUSÕES}

A temperatura inicial de oxidação da esfalerita presente no concentrado estudado foi de $680^{\circ} \mathrm{C}$ e a formação de sulfato de zinco pôde ser evidenciada durante a oxidação do mesmo. A pirita presente no concentrado foi oxidada em temperaturas inferiores àquelas encontradas para a esfalerita $\left(400^{\circ} \mathrm{C}\right)$ e os resultados sugerem que as etapas de oxidação dessa fase mineral estão de acordo com aquelas propostas por J. G. Dunn et. al [5].

Duas fases majoritárias, óxido de zinco e ferrita de zinco, foram detectadas no produto de oxidação obtido e esse produto formado é bastante poroso. Bons ajustes foram obtidos para os dados experimentais utilizando-se dos métodos isoconversionais integrais OFW, KAS e MNL. Os valores de energia de ativação aparente encontrados para os diferentes métodos variaram com o grau de conversão, mas não foram diferentes entre si em mais do que $2 \%$. Dessa forma, os valores encontrados de energia de ativação mostram que esse é um sistema complexo e que a etapa lenta do processo é uma etapa química e não uma etapa de transporte.

\section{Agradecimentos}

Os autores gostariam de agradecer ao Conselho Nacional do Desenvolvimento Científico e Tecnológico (CNPq), à Fundação de Amparo à Pesquisa do Estado de Minas Gerais (FAPEMIG), à Coordenação de Aperfeiçoamento de Pessoal de Nível Superior (CAPES), à Financiadora de Estudos e Projetos (FINEP) e a UFOP pelo apoio financeiro.

\section{REFERÊNCIAS}

I Dimitrov RI, Moldovanskab N, Bonevc IK, Zivkovicd Z. Oxidation of marmatite. Thermochimica Acta. 2000;362:|45-|5|.

2 Nicolov S, Boyanov B, Moldovanska N, Dimitrov R. Mössbauer spectroscopy study on the oxidation of sulfide zinc concentrate rich in marmatite. Thermochimica Acta. 200I;380:37-4I.

3 Boyanov BS, Dimitrov RI, Zivcovic CD. Thermal behaviour of low-quality zinc sulphide concentrate. Thermochimica Acta. 1997;296:123-128.

4 Dunn JG. The oxidation of sulphide minerals. Thermochimica Acta. 1997;300:127-139.

5 Dunn JG, O'Connor BH. The effect of experimental variables on the mechanism of the oxidation of pyrite: Part 2. Oxidation of particles of size 90-125 $\mu \mathrm{m}$. Thermochimica Acta. 1989; I 55: I35- 149.

6 Dimitrov RI, Bonev I. Mechanism of zinc sulphide oxidation. Thermochimica Acta. 1986; 106:9-25.

7 Iliyas I, Hawboldt K, Khan F. Advanced kinetics for calorimetric techniques and thermal stability screening of sulfide minerals. Thermochimica Acta. 2010;50:35-45.

8 Vyazovkin S. Handbook of thermal analysis and calorimetry. In: Parameshwaran R, Sarı A, Jalaiah N, Karunakaran R. Applications of thermal analysis to the study of phase-change materials. Oxford: Elsevier; 2008. p. 503-538.

9 Senum GI, Yang RTJ. Rational approximations of the integral of the Arrhenius function. Journal of Thermal Analysis. 1977; I I:445-447.

10 Vyazovkin S, Dollimore DJ. Linear and nonlinear procedures in isoconversional computations of the activation energy of nonisothermal reactions in solids. Chemical Information and Computer Science. 1996;36:42-45.

I I Vyazovkin S. A unified approach to kinetic processing of nonisothermal data. International Journal of Chemical Kinetics. 1996;28:95-101.

I2 Levenspiel O. Chemical reaction engineering. 3rd ed. New York: John Wiley \& Sons; 1999 [citado em 2018 janeiro 16]. Disponível em: https://4lfonsina.files.wordpress.com/20 I 2/ I //levenspiel-chemical-reaction-engineering.pdf

13 Vyazovkin S, Burnham AK, Criado JM, Maqueda LAP, Popescu C, Sbirrazzuoli N. ICTAC Kinetics Committee recommendations for performing kinetic computations on thermal analysis data. Thermochimica Acta. 20 I I;520: I- I9.

Recebido em: 08 Mar. 2018

Aceito em: 31 Ago. 2018

Tecnol. Metal. Mater. Miner., São Paulo, v. I6, n. 2, p. 142-149, abr./jun. 2019 